\title{
Electric Warmer
}

National Cancer Institute

\section{Source}

National Cancer Institute. Electric Warmer. NCI Thesaurus. Code C112509.

A device that converts electricity to heat. 